\title{
Total syntheses of 11-deoxytetrodotoxin and 8,11-dideoxytetrodotoxin*
}

\author{
Toshio Nishikawa, Daisuke Urabe, Kazumasa Yoshida, \\ Tomoko Iwabuchi, Masanori Asai, and Minoru Isobe
}

\begin{abstract}
Laboratory of Organic Chemistry, Graduate School of Bioagricultural Sciences, Nagoya University, Chikusa, Nagoya 464-8601, Japan
\end{abstract}

Abstract: 11-Deoxytetrodotoxin and 8,11-dideoxytetrodotoxin were synthesized from the same intermediate in the enantiomerically pure form.

\section{INTRODUCTION}

Tetrodotoxin [1] (TTX, 1), originally isolated from puffer fish, is one of the most famous marine natural products because of its novel structure and potent biological activity. Since TTX was revealed to inhibit sodium ion influx through voltage-dependent sodium channels, this toxin has been employed as an indispensable tool in neurophysiological experiments. There are many unresolved issues associated with TTX, such as the details of the bound structure to the $\mathrm{Na}^{+}$channel protein, and accumulation/detoxification mechanisms in puffer fish; the biosynthesis and actual biological functions of this toxin are also only partially understood. In order to study these problems on a molecular level, the total chemical synthesis of molecular probes such as labeled TTX is desirable, because the derivatization of naturally occurring TTX is quite difficult. In spite of many attempts to synthesize this molecule, the sole total synthesis of the racemate was reported by Kishi and coworkers in 1972 [2]. In the course of our synthetic studies of TTX and its analogs, we reported synthesis of (-)-5,11-dideoxytetrodotoxin 4 in 1999 [3]. However, that synthetic sample showed little biological activity, probably because it lacked the characteristic ortho ester. We disclose herein the successful syntheses of two TTX analogs, 11-deoxytetrodotoxin $\mathbf{2}$ and 8,11-dideoxytetrodotoxin 3, both of which have ortho ester functionality.

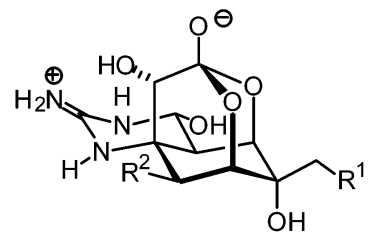

tetrodotoxin (TTX, 1) $\mathrm{R}^{2}=\mathrm{OH} \quad \mathrm{R}^{1}=\mathrm{OH}$

11-deoxyTTX (2) $\quad \mathrm{R}^{2}=\mathrm{OH} \quad \mathrm{R}^{1}=\mathrm{H}$

8,11-dideoxyTTX (3) $R^{2}=H \quad R^{1}=H$

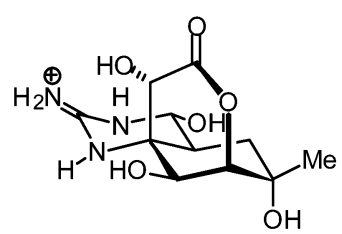

5,11-dideoxyTTX (4)

\footnotetext{
* Pure Appl. Chem. 75, 141-419 (2003). An issue of reviews and research papers based on lectures presented at the $23^{\text {rd }}$ IUPAC International Symposium on the Chemistry of Natural Products, Florence, Italy, 28 July-2 August 2002 on the theme of natural products.

${ }^{\ddagger}$ Corresponding author
} 


\section{SYNTHETIC PLAN FOR DEOXYTETRODOTOXINS}

We plan to synthesize a variety of natural and unnatural TTX analogs from the same intermediate $\mathbf{9}$, which was synthesized in 9 steps from levoglucosenone as a chiral starting material [4] (Scheme 1). 11-Deoxytetrodotoxin $\mathbf{2}$ is interconvertible to its 4,9-anhydro derivative $\mathbf{5}$ under acidic conditions, and the ortho ester group is equivalent to $\delta$-hydroxyl lactone. The cyclic guanidine could be synthesized from diacetylguanidine and acetonide in $\mathbf{6}$. The intramolecular acetal was designed for the protection of the labile C9 position as well as for successful global deprotection at the final stage of synthesis. During the course of our TTX project, we developed a new method for guanidine synthesis from trichloroacetamide [5]; that methodology would be applied to the synthesis of $\mathbf{6}$. These analyses led us to identify lactone $\mathbf{7}$ as an important intermediate. The lactone $\mathbf{7}$ was then retrosynthesized to the vinyl epoxide 8. Finally, this highly oxygenated cyclohexane could be synthesized from the common intermediate 9. Thus, the synthesis started with regio- and stereoselective hydroxylation of $\mathbf{9}$.
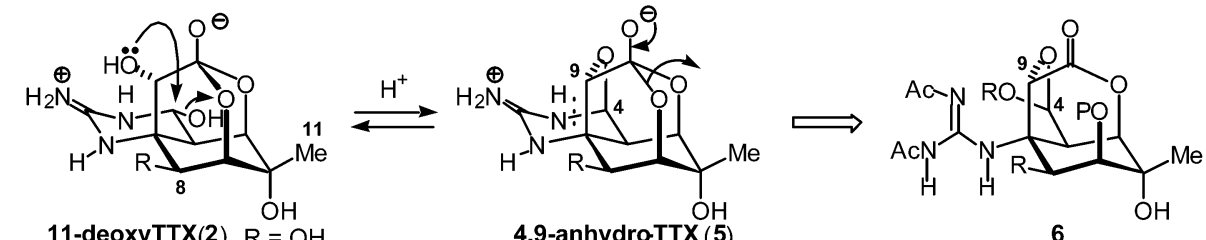

8,11-dideoxyTTX(3) $\mathrm{R}=\mathrm{H}$
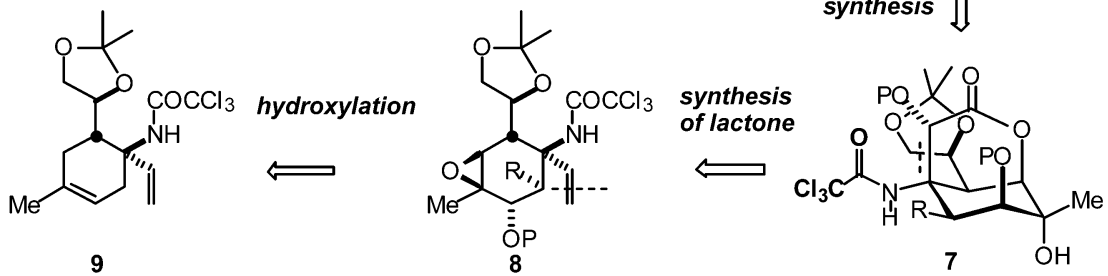

Scheme 1 Synthetic plan for 11-deoxy and 8,11-dideoxytetrodotoxin.

In the synthesis of (-)-5,11-dideoxytetrodotoxin 4, we found a novel hydroxylation of the C8 position, as shown in Scheme 2. The common intermediate $\mathbf{9}$ was brominated to $\mathbf{1 0}$, which was treated with 1,5-diazabicyclo[5.4.0] undecene-5 (DBU) in dimethylformamide (DMF) to give oxazoline $\mathbf{1 1}$ in one operation. Acid hydrolysis of $\mathbf{1 1}$ gave allylic alcohol 12. This product serves as a suitable synthetic

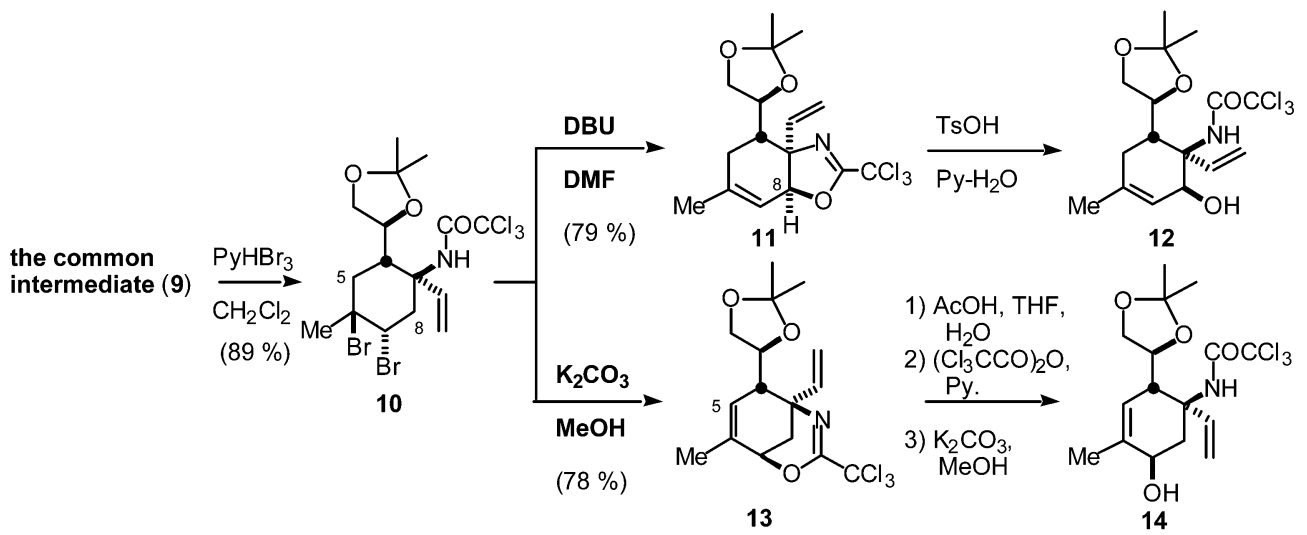

Scheme 2 Novel hydroxylation with neighboring group participation. 
intermediate not only for $\mathbf{4}$, but also for 11-deoxytetrodotoxin $\mathbf{2}$. In sharp contrast, the same dibromide 10 was treated with $\mathrm{K}_{2} \mathrm{CO}_{3}$ in $\mathrm{MeOH}$ to give bicyclic iminoether 13, which was transformed to allylic alcohol 14 in three steps. The product 14 is a suitable precursor for 8,11-dideoxytetrodotoxin 3 . The different reactivity can be rationalized by the selective dehydrobromination from $\mathbf{1 0}$ under the different conditions. When DMF was employed as a solvent, DBU abstracted a proton on the nitrogen of trichloroacetamide group, and then a generated oxygen anion abstracted the closest, but the most hindered proton at $\mathrm{C} 8$ to give undetectable allylic bromide, which then underwent an $\mathrm{S}_{\mathrm{N}} 2^{\prime}$ reaction with trichloroacetamide to give 11. Under protic conditions with methanol, the base abstracted the less hindered proton at $\mathrm{C} 5$ to give a different allylic bromide. A subsequent $\mathrm{S}_{\mathrm{N}} 2$ reaction with trichloroacetamide gave $\mathbf{1 3}$.

\section{TOTAL SYNTHESIS OF 11-DEOXYTETRODOTOXIN [6]}

The allylic alcohol 12 was epoxidized with $m$-chloro peroxybenzoic acid (MCPBA) to give $\beta$-epoxide, which was then converted to allylic alcohol 16 with $\mathrm{Ti}(\mathrm{O} i-\mathrm{Pr})_{4}$ (Scheme 3). Since both of the diol configurations were the opposite of those of 11-deoxytetrodotoxin, we attempted a simultaneous inversion of the configuration through diketone $\mathbf{1 7}$ obtained from $o$-iodosoxybenzoic acid (IBX) oxidation of $\mathbf{1 6}$. Although the stereoselective reduction of $\mathbf{1 7}$ proved to be very difficult, we fortunately found that a twostep reduction gave the desired diol $\mathbf{1 9}$ in a good overall yield. First, the reduction was conducted with $\mathrm{LiAlH}(\mathrm{O} t-\mathrm{Bu})_{3}$ in the presence of $\mathrm{LiBr}$ to give a mixture of $\alpha$-hydroxyl unsaturated ketone $\mathbf{1 8}$ and the desired diol 19. The crude products, without purification, were subjected to Luche's reduction at $-78{ }^{\circ} \mathrm{C}$ to give the desired diol 19 in a ca. $80 \%$ isolated yield from diol $\mathbf{1 6}$.

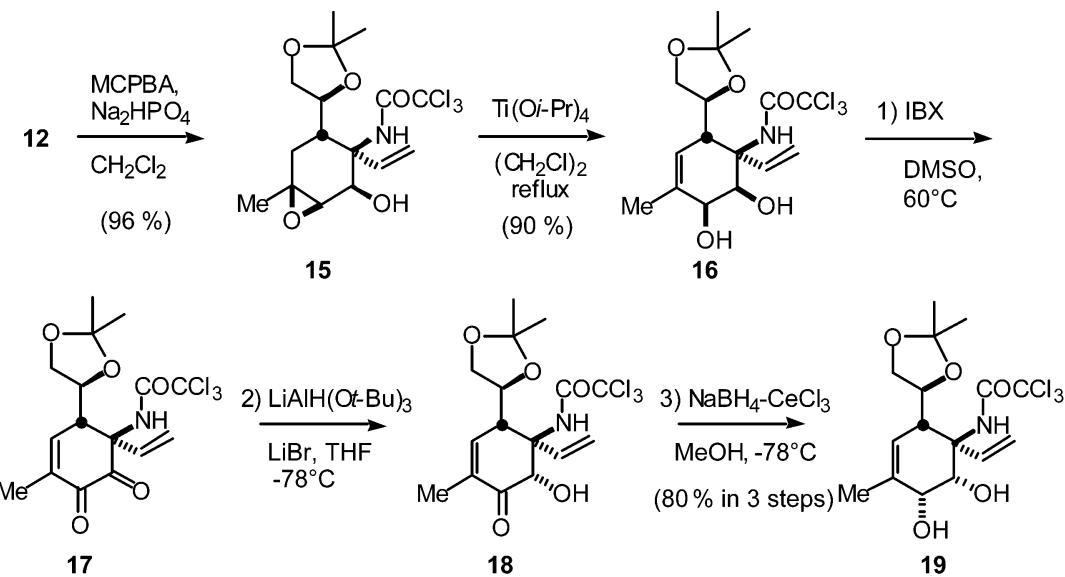

Scheme 3

After the two hydroxyl groups of $\mathbf{1 9}$ were protected as trimethylsilyl (TMS) ether, the trisubstituted olefin was epoxidized with MCPBA at room temperature (rt) to give $\beta$-epoxide $\mathbf{2 0}$ in a high yield (Scheme 4). Interestingly, the protective group of the diol was found to be critical for epoxidation; the unprotected diol 19 and dibenzyl ether of 19 did not react at all with MCPBA at rt.

The next task was transformed into $\alpha$-hydroxylcarboxylic acid as a precursor for a lactone intermediate such as 7. However, the vinyl group was very inert in the synthesis of 5,11-dideoxytetrodotoxin, probably due to the severe steric hindrance around this alkene. Consequently, the vinyl group was ozonized to aldehyde 21, which was reacted with magnesium acetylide to give adduct 22 in good diastereomeric selectivity. The major isomer was easily separated, and transformed to $\mathbf{2 3}$ in two steps including acetylation and selective desilylation of silylacetylene. Unexpectedly, the acetylenic group 


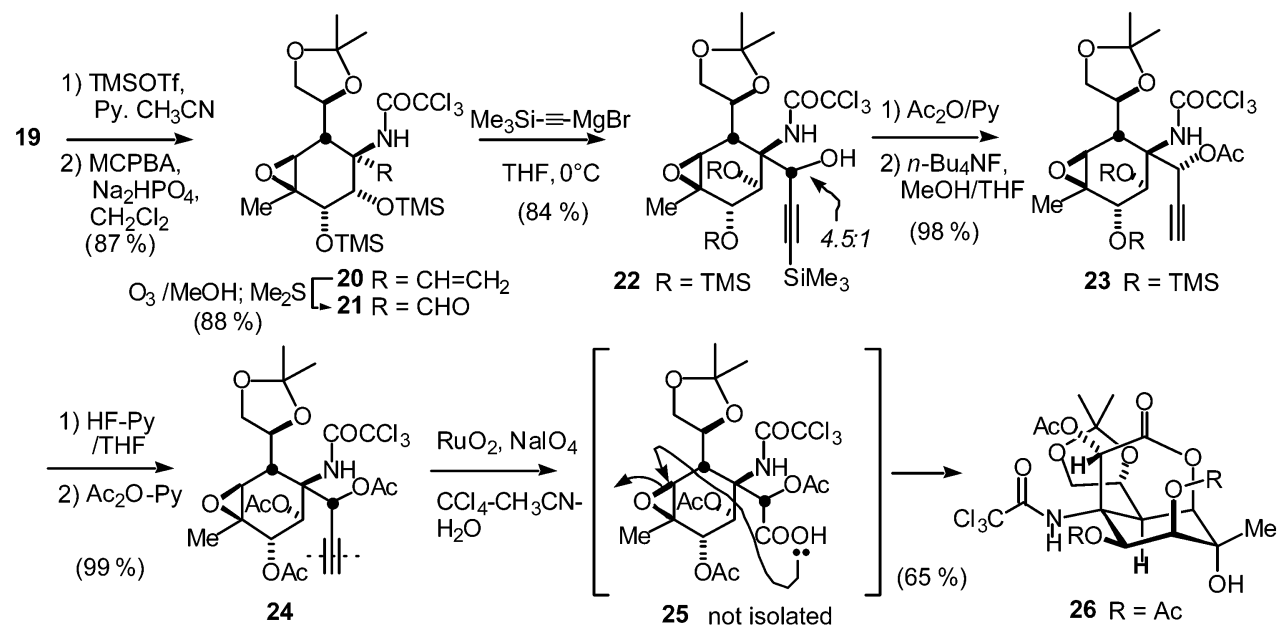

Scheme 4 Synthesis of the lactone intermediate.

did not react at all with $\mathrm{RuO}_{4}$. We reasoned that this extraordinarily low reactivity might be due to the steric hindrance caused by the TMS ethers and trichloroacetamide. We therefore decided to use smaller protective groups. Bis-TMS groups were converted to the corresponding acetate $\mathbf{2 4}$ in 2 steps, including desilylation with HF-pyridine followed by acetylation. We were please to find that the acetylenic group was oxidized with $\mathrm{RuO}_{4}$ to give carboxylic acid $\mathbf{2 5}$, which spontaneously opened the epoxide under these conditions to give lactone $\mathbf{2 6}$.

In the synthesis of 5,11-dideoxytetrodotoxin, we encountered serious problems such as epimerization and oxidation at the C9 position. To overcome these problems, we designed intramoleculer mixed acetal 28, which was synthesized from lactone $\mathbf{2 6}$ in three steps (Scheme 5). Product $\mathbf{2 8}$ was set for the guanidinylation that was developed for the synthesis of TTXs. tert-Alcohol $\mathbf{2 8}$ was acetylated and then heated with sodium carbonate at ca. $100{ }^{\circ} \mathrm{C}$ to give an unstable isocyanate, which was captured with benzylamine to give benzylurea 29. The urea was dehydrated with $\mathrm{Ph}_{3} \mathrm{P}$ and $\mathrm{CBr}_{4}$ to give the benzylcarbodiimide 30. Addition of benzylamine to the carbodiimide was best accomplished with benzylamine hydrochloride under pyridine reflux conditions to give dibenzylguanidinium salt, which was isolated as the corresponding acetate $\mathbf{3 1}$.
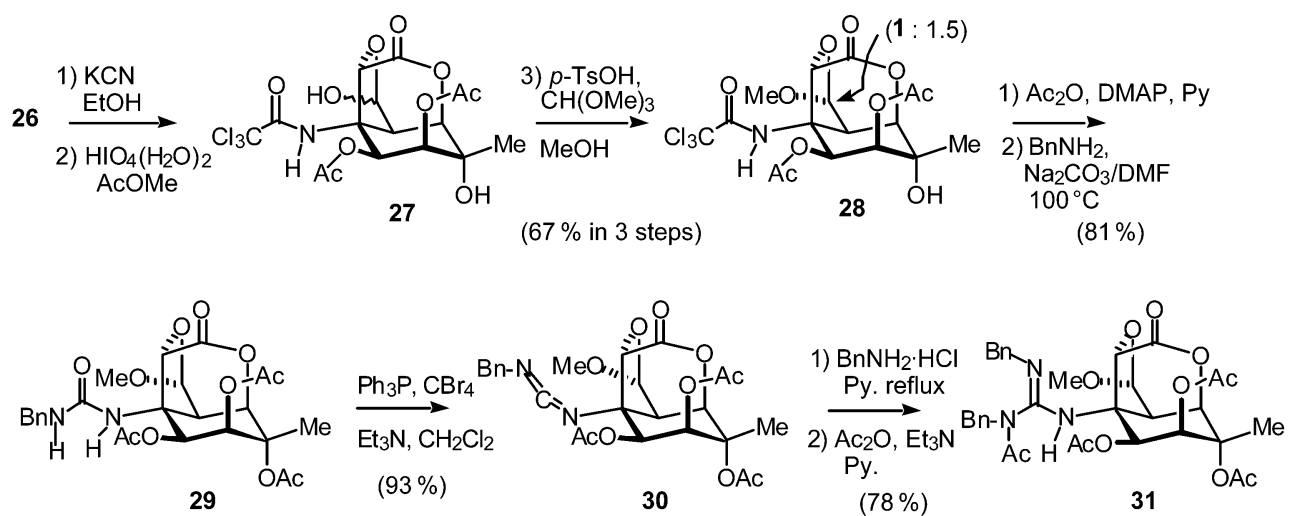

Scheme 5 
The remaining problem for the total synthesis was deprotection of 31. The benzyl groups of guanidine were hydrogenolyzed in acetic anhydride to give the corresponding acetate 32 (Scheme 6). The acetyl groups were removed with aqueous ammonia, and finally the acetal was hydrolyzed with aqueous trifluoroacetic (TFA) to furnish 11-deoxytetrodotoxin $\mathbf{2}$ and its 4,9-anhydro derivative $\mathbf{3 3}$.

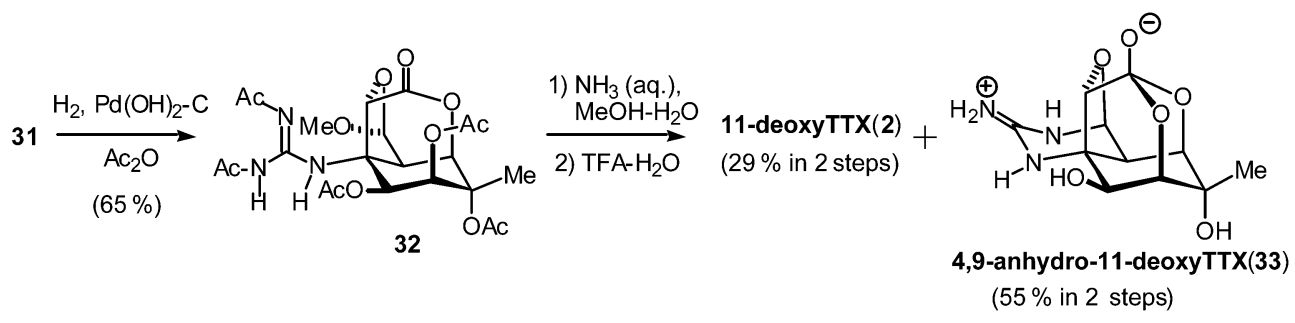

Scheme 6 Total synthesis of 11-deoxytetrodotoxin.

\section{SYNTHESIS OF 8,11-DIDEOXYTETRODOTOXIN [7]}

The major purpose of the synthesis of 8,11-dideoxytetrodotoxin 3, an unnatural TTX analog, was to clarify the biological role of the 8-hydroxyl group. Since 8-deoxytetrodotoxin analogs have not been found among natural sources, little information is available on the structure-activity relationship of this hydroxyl group. The synthesis of 8,11-dideoxytetrodotoxin $\mathbf{3}$ was expected to be very similar to the synthesis of 11-deoxytetrodotoxin 2, because of the structural similarity of these molecules. According to the synthesis of $\mathbf{2}$, fully protected 8,11-dideoxytetrodotoxin $\mathbf{3 8}$ was synthesized from 14, which was prepared from the common intermediate 9 (Scheme 7); allylic alcohol 14 was epoxidized with MCPBA and then the configuration of the hydroxyl group was inverted to give 34. In this case, the addition of lithium acetylide to the aldehyde obtained from ozonolysis of $\mathbf{3 4}$ gave propargyl alcohol $\mathbf{3 5}$ with the undesired configuration. Thus, the hydroxyl group was again inverted by oxidation-reduction in a highly stereoselective manner. Oxidative cleavage of the alkyne with $\mathrm{RuO}_{4}$ was followed by treatment with acid to give lactone 36, which was transformed to intramolecular mixed acetal 37. Trichloroacetamide $\mathbf{3 7}$ was transformed to diacetylguanidine $\mathbf{3 8}$ according to the synthesis of $\mathbf{3 2}$. The same deprotection procedure employed in the synthesis of 11-deoxytetrodotoxin 2 should be applicable to the deprotection of $\mathbf{3 8}$. However, $\mathbf{3 8}$ showed completely different behavior under identical deprotection conditions.

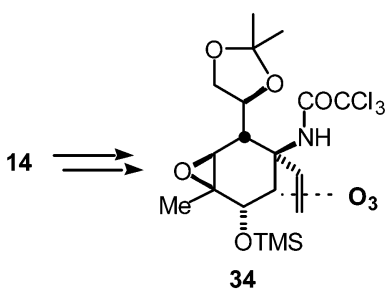

34

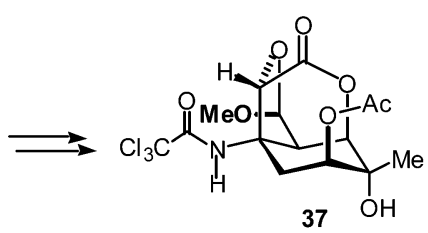<smiles>C=CC#N</smiles>

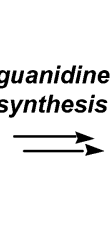

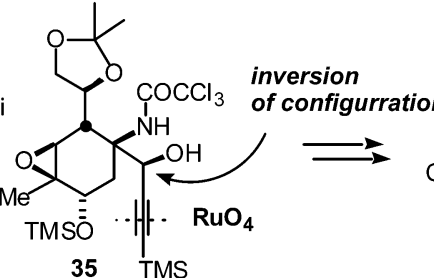

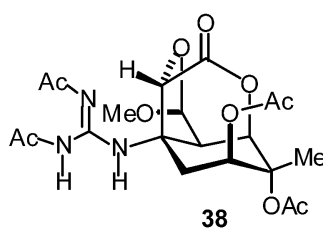

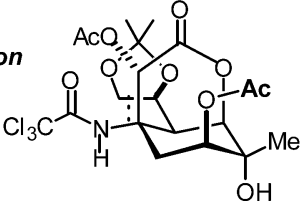

36

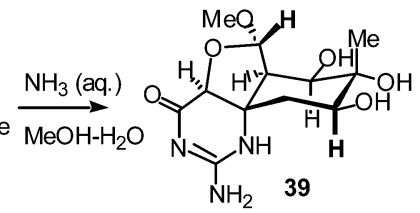

Scheme 7 
When the tetraacetate $\mathbf{3 8}$ was treated with aqueous ammonia, we did not detect any desired products in the crude mixture. The structure of the product was assumed to be $\mathbf{3 9}$, as based on 2D NMR analysis. Further acid treatment gave neither 8,11-dideoxytetrodotoxin 3 nor its 4,9-anhydro derivative. To overcome this problem, we modified our original synthetic plan as shown in Scheme 8.
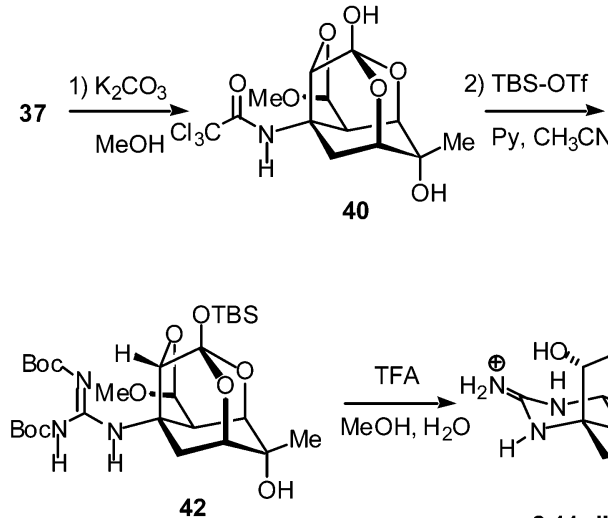

(85\% in 2 steps)

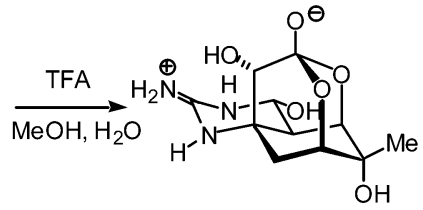

8,11-dideoxyTTX(3)

(43\%)

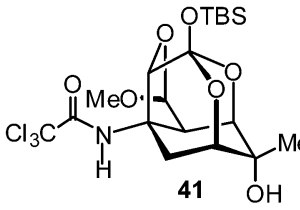

(64\% in 2 steps)

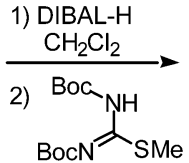

$\mathrm{HgCl}_{2}, \mathrm{Et}_{3} \mathrm{~N}, \mathrm{DMF}$

Scheme 8 Synthesis of 8,11-dideoxytetrodotoxin.

Fortunately, it was found that when 37 was treated with $\mathrm{K}_{2} \mathrm{CO}_{3}$ in $\mathrm{MeOH}$, the reaction gave ortho ester 40, which was protected as TBS ether 41. Reductive deprotection of the trichloroacetamide 41 with diisobutylaluminum hydride (DIBAL-H) afforded amine, which was treated with Boc-protected isothiourea in the presence of mercuric salt to give Boc-protected guanidine $\mathbf{4 2}$ in a good overall yield. To our delight, all of the protective groups were removed with TFA in water to give 8,11-dideoxytetrodotoxin 3 and 4,9-anhydro-8,11-dideoxytetrodotoxin $\mathbf{4 3}$. We are currently determining the biological activity of $\mathbf{3}$.

In summary, we have achieved the asymmetric syntheses of 11-deoxytetrodotoxin 2 and 8,11-dideoxytetrodotoxin $\mathbf{3}$ from a common intermediate $\mathbf{9}$. The synthesis of $\mathbf{2}$ is the first asymmetric total synthesis of a naturally occurring TTX analog. In the synthesis of $\mathbf{3}$, we developed an efficient guanidine installation through an ortho ester intermediate. Total synthesis of TTX $\mathbf{1}$ is currently under way.

\section{ACKNOWLEDGMENTS}

We thank Profs. M. Yotsu-Yamashita and T. Yasumoto for providing the NMR data of the natural products. This work was financially supported by JSPS-RFTF; a Grant-in-Aid for Scientific Research from the Ministry of Education, Science, and Culture of Japan; the Pfizer's Award in Synthetic Organic Chemistry; and the Mitsubishi Chemical Corporation Fund.

\section{REFERENCES}

1. (a) T. Goto, Y. Kishi, S. Takahashi, Y. Hirata. Tetrahedron 21, 2059 (1965); (b) K. Tsuda, S. Ikuma, M. Kawamura, R. Tachikawa, K. Sakai, C. Tamura, O. Amakasu. Chem. Pharm. Bull. 12, 1357 (1964); (c) R. B. Woodward. Pure. Appl. Chem. 9, 49 (1964).

2. Y. Kishi, M. Aratani, T. Fukuyama, F. Nakatsubo, T. Goto, S. Inoue, H. Tanino, S. Sugiura, H. Kakoi. J. Am. Chem. Soc. 94, 9217, 9219 (1972). 
3. T. Nishikawa, M. Asai, N. Ohyabu, N. Yamamoto, M. Isobe. Angew. Chem., Int. Ed. 38, 3081 (1999); M. Asai, T. Nishikawa, N. Ohyabu, N. Yamamoto, M. Isobe. Tetrahedron 57, 4543 (2001).

4. T. Nishikawa, M. Asai, N. Ohyabu, N. Yamamoto, Y. Fukuda, M. Isobe. Tetrahedron 57, 3875 (2001).

5. T. Nishikawa, N. Ohyabu, N. Yamamoto, M. Isobe. Tetrahedron 55, 4325 (1999).

6. T. Nishikawa, M. Asai, M. Isobe. J. Am. Chem. Soc. 124, 7847 (2002).

7. T. Nishikawa, D. Urabe, K. Yoshida, T. Iwabuchi, M. Asai, M. Isobe. Org. Lett. 4, 2679 (2002). 